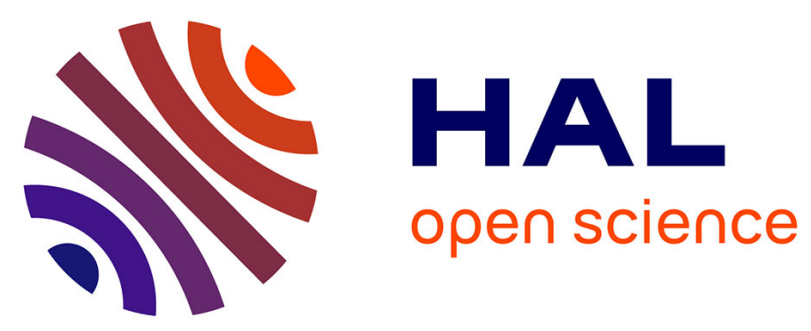

\title{
Fast acoustic streaming in standing waves: Generation of an additional outer streaming cell
}

Ida Reyt, Hélène Bailliet, Solène Moreau, Jean-Christophe Valière, Diana G. Baltean Carlès, Catherine Weisman, Virginie Daru

\section{- To cite this version:}

Ida Reyt, Hélène Bailliet, Solène Moreau, Jean-Christophe Valière, Diana G. Baltean Carlès, et al.. Fast acoustic streaming in standing waves: Generation of an additional outer streaming cell. Journal of the Acoustical Society of America, 2013, 134 (3), pp.1791-1801. 10.1121/1.4817888 . hal-01066956

\section{HAL Id: hal-01066956 https://hal.science/hal-01066956}

Submitted on 9 Oct 2017

HAL is a multi-disciplinary open access archive for the deposit and dissemination of scientific research documents, whether they are published or not. The documents may come from teaching and research institutions in France or abroad, or from public or private research centers.
L'archive ouverte pluridisciplinaire HAL, est destinée au dépôt et à la diffusion de documents scientifiques de niveau recherche, publiés ou non, émanant des établissements d'enseignement et de recherche français ou étrangers, des laboratoires publics ou privés. 


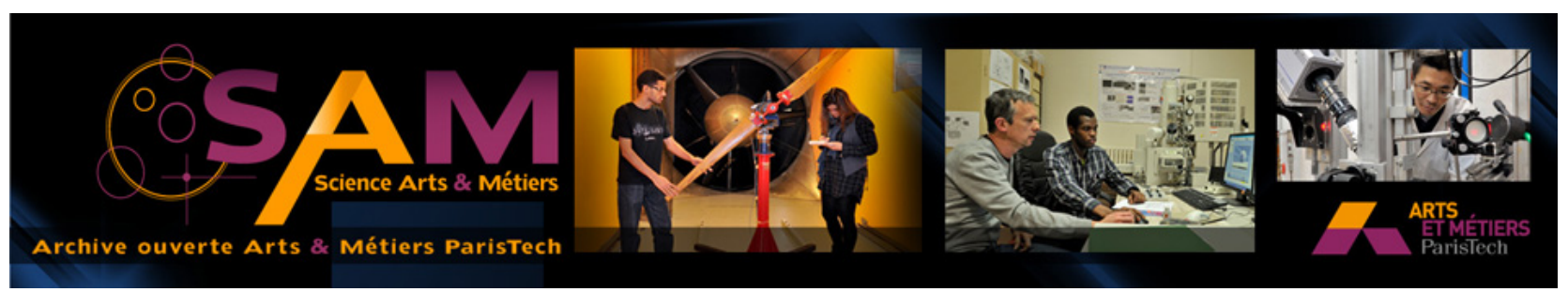

\section{Science Arts \& Métiers (SAM)}

is an open access repository that collects the work of Arts et Métiers ParisTech researchers and makes it freely available over the web where possible.

This is an author-deposited version published in: http://sam.ensam.eu

Handle ID: .http://hdl.handle.net/10985/8595

\section{To cite this version :}

Ida REYT, Hélène BAILLIET, Solène MOREAU, Jean-Christophe VALIERE, Diana BALTEANCARLES, Catherine WEISMAN, Virginie DARU - Fast acoustic streaming in standing waves : Generation of an additional outer streaming cell - Journal of the Acoustical Society of America Vol. 134, n³, p.1791-1801- 2013 


\title{
Fast acoustic streaming in standing waves: Generation of an additional outer streaming cell
}

\author{
Ida Reyt \\ LIMSI-CNRS UPR 3251, \\ Bât. 508 rue John Von Neumann, \\ Campus Univ. Paris Sud, \\ 91403 Orsay, \\ France
}

Hélène Bailliet, Solène Moreau, ${ }^{\text {a) }}$ and Jean-Christophe Valière Institut Pprime, CNRS - Université de Poitiers - ENSMA, Département Fluides - Thermique - Combustion, ENSIP 6 rue Marcel Doré Bât. B17 - BP 633, 86022 Poitiers Cedex, France

Diana Baltean-Carlès ${ }^{\mathrm{b})}$ and Catherine Weisman ${ }^{\mathrm{b})}$

$$
\begin{gathered}
\text { UPMC Paris06 UFR 919, } \\
4 \text { Place Jussieu, } \\
75252 \text { Paris Cedex 05, } \\
\text { France }
\end{gathered}
$$

Virginie Daru ${ }^{\mathrm{b}}$

Arts et Métiers ParisTech Lab. DynFluid, 151 bd de l'hôpital, 75013 Paris, France 
(Dated: July 8, 2013) 


\begin{abstract}
Rayleigh streaming in a cylindrical acoustic standing waveguide is studied both experimentally and numerically, for nonlinear Reynolds numbers from 1 to $30\left(R e_{N L}=\left(\frac{U_{0}}{c_{0}}\right)^{2}\left(\frac{R}{\delta_{\nu}}\right)^{2}\right.$ with $U_{0}$ the acoustic velocity amplitude at the velocity antinode, $c_{0}$ the speed of sound, $R$ the tube radius and $\delta_{\nu}$ the acoustic boundary layer thickness). Streaming velocity is measured by means of Laser Doppler Velocimetry (LDV) in a cylindrical resonator filled with air at atmospheric pressure at high intensity sound levels. The compressible Navier-Stokes equations are solved numerically with high resolution finite difference schemes. The resonator is excited by shaking it along the axis at imposed frequency. Results of measurements and of numerical calculation are compared with results given in the literature and with each other. As expected, the axial streaming velocity measured and calculated agrees reasonably well with the slow streaming theory for small $R e_{N L}$ but deviates significantly from such predictions for fast streaming $\left(R e_{N L}>1\right)$. Both experimental and numerical results show that when $R e_{N L}$ is increased, the center of the outer streaming cells are pushed toward the acoustic velocity nodes until counter-rotating additional vortices are generated near the acoustic velocity antinodes.
\end{abstract}

PACS numbers: 43.25.Nm,43.25.Gf 


\section{INTRODUCTION}

Rayleigh streaming refers to the second order mean velocity that is generated by viscous effects related with the interaction of a solid surface and an acoustic wave in a fluid. In thermoacoustic devices, acoustic streaming results in convected heat flow that can reduce the efficiency of the devices. Because the power density of a thermoacoustic device is roughly proportional to the square of the acoustic pressure amplitude, the study of high acoustic amplitude phenomena is important to the field of thermoacoustics. A better understanding and characterization of the corresponding so-called "fast" Rayleigh streaming is therefore useful to assess its impact on the efficiency of thermoacoustic devices.

The reference solution derived by Rayleigh ${ }^{1}$ concerns streaming generated by a standing wave between two infinite, widely separated plates. Together with further studies by Nyborg $^{2}$ and Westervelt ${ }^{3}$, this work yielded the so-called RNW streaming theory. For a better application to thermoacoustics, this theoretical description has evolved in order to refine thermal effect description and to remove the restrictions to wide channels and to slow streaming. Concerning the first two points, during the last decades, thermoacoustic applications have motivated several theoretical works to take into account thermal effects ${ }^{4,5}$ and/or to remove the restriction to large channels ${ }^{6,7}$. However, all these theoretical developments were restricted to slow streaming where fluid inertia is neglected. The dimensionless number characterizing the inertial effects with respect to viscous effects is the nonlinear Reynolds number $R e_{N L}$ defined by Menguy and Gilbert ${ }^{8}$. For slow streaming the nonlinear Reynolds number is such that $R e_{N L}<<1$. The influence of fluid inertia was studied only by Menguy and Gilbert and for $R e_{N L}$ up to about 4 only; using a perturbation method with asymptotic expansions followed by time-averaging, they developed boundary layer streaming equations, matched with nonlinear streaming equations in the main acoustic field, thus demonstrating

a) Current address:Laboratoire Roberval CNRS 6253, Université de Technologie de Compiègne BP 20529, 60205 Compiègne Cedex, France

b) Also at LIMSI-CNRS UPR 3251, 91403 Orsay, France 
a noticeable distortion of the streaming velocity field.

Previous simulations of acoustic streaming in the linear regime were conducted by solving the flow dynamics between two plates inside a thermoacoustic refrigerator ${ }^{9}$. More recently, Aktas and Farouk ${ }^{10}$ performed numerical simulations in the linear and nonlinear streaming regimes, solving the full compressible Navier-Stokes equations in the plane case with a FCT scheme. The acoustic excitation was provided by a moving wall. Their results show a distortion of the streaming field and the existence of multiple streaming cells for small values of $R e_{N L}$, even for values less than 1. In their analysis, the authors correlate the existence of the nonlinear streaming regime with the values of the aspect ratio of the waveguide and of a different streaming Reynolds number $\left(R e_{s}=U_{0}^{2} /(\nu \omega), \omega\right.$ being the angular frequency). When their results are analyzed as a function of $R e_{N L}$, the tendencies depicted are in contradiction with experiments ${ }^{11,12}$. One of the goals of the present paper is to present direct numerical simulations of the Navier-Stokes equations in the cylindrical case using a recently developed high resolution numerical scheme ${ }^{13}$ tested successfully in several cases (including acoustics and streaming ${ }^{14,15}$ ). In the present simulations, the acoustic wave is excited by shaking the entire resonator and there are no restrictions on the value of the $R e_{N L}$

With respect to experimental studies, thanks to improving techniques in fluid dynamics, measurements of the streaming in a standing wave guide have been performed recently ${ }^{11,16-19}$. Experimental works by Sharpe, Greated and collaborators ${ }^{16}$ and by Thompson and Atchley ${ }^{19}$ first allowed quantitative study of streaming velocity in the core region on the basis of Particle Image Velocimetry (PIV) or Laser Doppler Velocimetry (LDV) measurements. Moreau et $a l .{ }^{11}$ first performed LDV measurements of streaming in both the core and the near wall region to characterize inner streaming vortices. Their measurements were performed for $R e_{N L}$ up to 140 but they mainly focused on inner streaming cells evolution. In their experimental study, Thompson and Atchley ${ }^{19}$ considered streaming for $R e_{N L}$ up to 20 and found that the streaming fields is distorted for high nonlinear Reynolds number in correlation with the increasing temperature gradient along a streaming cell. Nabavi et al. ${ }^{12}$ analyzed stream- 
ing in a square channel on the basis of PIV measurements; they found that for $R e_{N L}$ up to 25, streaming cells are regular and correspond to "classical" streaming flow, whereas when $R e_{N L}$ exceeds 25, streaming deforms to irregular and complex shaped patterns. Another goal of the present work is to present results of measurements of the streaming velocity field generated by an acoustic standing wave in a cylindrical resonator by means of LDV from low to high acoustic amplitudes (up to $R e_{N L}$ greater than 25).

In the present study, numerical and experimental results are successfully compared with available theoretical expectations in the linear regime. For this purpose, a slow streaming analytical model that has been developed previously ${ }^{6}$ for the calculation of Rayleigh streaming in a cylindrical waveguide is used. Then the analysis of the nonlinear regime is conducted for the first time with a coupled numerical/experimental approach on a cylindrical geometry, and the results are confronted. Both simulations and experiments show that streaming at high $R e_{N L}$ is associated with additional outer streaming cells appearing at a critical value of this number of about $R e_{N L}=30$.

Section II presents the different methods for the calculation of streaming that are used or referred to in this paper (Direct numerical method, work by Bailliet et $a l^{6}$, and work by Menguy and Gilbert ${ }^{8}$ ). The dimensionless numbers used to describe the problem of Rayleigh streaming in a standing wave guide are presented as it is necessary to consider the respective values of these numbers in order to validate the comparison between numerical and experimental results. In Section III, experimental set up and procedure are presented. Section IV presents the numerical method used to calculate streaming velocity. Finally in Section $\mathrm{V}$, these numerical and experimental results are compared with each other and with the available literature.

\section{FUNDAMENTAL EQUATIONS AND NON-DIMENSIONAL ANALYSIS}

All the methods used to calculate the streaming flow in this paper are based on the fundamental equations that describe an acoustic wave propagation in the laminar regime 
along the $z$ axis, in a cylindrical tube of radius $R$ filled with air at atmospheric pressure. The ratio of the wavelength to the transverse dimension is supposed to be large so that the wave is plane. Air is considered as a Newtonian compressible fluid and there is no mean flow apart from acoustic streaming. Gravity is neglected. Neglecting the bulk viscosity in comparison with the shear viscosity, these fundamental equations (Navier-Stokes equations) that describe the fluid motion are written as

$$
\left\{\begin{array}{l}
\frac{\partial \rho}{\partial t}+\nabla \cdot(\rho \mathbf{u})=0 \\
\frac{\partial(\rho \mathbf{u})}{\partial t}+\nabla \cdot(\rho \mathbf{u} \otimes \mathbf{u})+\nabla p=\nabla \cdot(\overline{\bar{\tau}}) \\
\frac{\partial(\rho E)}{\partial t}+\nabla \cdot(\rho E \mathbf{u}+p \mathbf{u})=\nabla \cdot(k \nabla T)+\nabla \cdot(\overline{\bar{\tau}} \mathbf{u})
\end{array}\right.
$$

where $\rho$ is the density, $p$ is the pressure, $\mathbf{u}$ is the flow velocity vector, $E=e+\frac{1}{2} \mathbf{u} \cdot \mathbf{u}$ is the total

energy, with $e=\frac{p}{(\gamma-1) \rho}$ the internal energy, $\gamma$ the specific heat ratio, $\overline{\bar{\tau}}=-\frac{2}{3} \mu(\nabla \cdot \mathbf{u}) \overline{\bar{I}}+2 \mu \overline{\bar{D}}$

the viscous stress tensor, with $\overline{\bar{I}}$ the identity tensor, $\overline{\bar{D}}=\frac{1}{2}\left(\nabla \mathbf{u}+\nabla \mathbf{u}^{T}\right)$ the strain tensor, $\mu$ the dynamic viscosity, $k$ the thermal conductivity. The gas is considered as a perfect gas obeying the state law $p=r_{g} \rho T$, where $T$ is the temperature and $r_{g}$ is the constant of the perfect gas (air in the present case).

\section{A. Non-dimensional analysis}

In the previous equations, there are 6 unknown physical variables, $\rho, \mathbf{u}(3$ components), $p$ and $T$ (the internal energy $e$ can be replaced by $\left(c_{p} / \gamma\right) T, c_{p}$ being the specific heat capacity at constant pressure) and 6 equations. The problem is written in a dimensionless form, using standard fluid mechanics scalings:

$$
\hat{\nabla}=\frac{\nabla}{L_{0}}, \quad \hat{t}=\frac{t}{t_{0}}, \quad \hat{\mathbf{u}}=\frac{\mathbf{u}}{U_{0}}, \quad \hat{p}=\frac{p}{p_{0}}, \quad \hat{\rho}=\frac{\rho}{\rho_{0}}, \quad \hat{T}=\frac{T}{T_{0}}, \quad \hat{\mu}=\frac{\mu}{\mu_{0}}, \quad \hat{k}=\frac{k}{k_{0}},
$$

where $L_{0}, t_{0}, U_{0}$ are reference scales for length, time and velocity (based on the acoustic propagation), and $p_{0}, \rho_{0}, T_{0}, \mu_{0}, k_{0}$ are scales for pressure, density, temperature, dynamic viscosity and conductivity (based on fluid properties at rest). In the context of acoustics, 
the adiabatic speed of sound is defined as $c_{0}=\sqrt{\left(\frac{\partial p}{\partial \rho}\right)_{s, 0}}$, also written as $c_{0}=\sqrt{\gamma \frac{p_{0}}{\rho_{0}}}$ using the first-order Taylor development of pressure. The characteristic acoustic time and length scales are given by $t_{0}=\frac{1}{\omega}, L_{0}=\frac{c_{0}}{\omega}$, where $\omega$ is the fundamental acoustic angular frequency. $U_{0}$ is a reference value of the acoustic velocity, for example the maximum amplitude of the fundamental component of the wave. With these reference scales, 3 independent dimensionless numbers are defined by rewriting the set of equations 1 : the acoustic Mach number $M=\frac{U_{0}}{c_{0}}$, the acoustic Reynolds number $R e=\frac{\rho_{0} c_{0}^{2}}{\mu_{0} \omega}$ and the Prandtl number $\operatorname{Pr}=\frac{\mu_{0} c_{p}}{k_{0}}$. The equations (1) in dimensionless form are indeed

$$
\left\{\begin{array}{l}
\frac{\partial \hat{\rho}}{\partial \hat{t}}+M \hat{\nabla} \cdot(\hat{\rho} \hat{\mathbf{u}})=0 \\
\frac{\partial(\hat{\rho} \hat{\mathbf{u}})}{\partial \hat{t}}+M \hat{\nabla} \cdot(\hat{\rho} \hat{\mathbf{u}} \otimes \hat{\mathbf{u}})+\frac{1}{\gamma M} \hat{\nabla} \hat{p}=\frac{1}{R e} \hat{\nabla} \cdot(\hat{\bar{\tau}}) \\
\frac{1}{\gamma} \frac{\partial(\hat{\rho} \hat{T})}{\partial \hat{t}}+M^{2}(\gamma-1) \frac{\partial\left(\hat{\rho} \frac{1}{2}|\hat{\mathbf{u}}|^{2}\right)}{\partial \hat{t}}+\frac{M}{\gamma} \hat{\nabla} \cdot(\hat{\rho} \hat{T} \hat{\mathbf{u}})+M^{3}(\gamma-1) \hat{\nabla} \cdot\left(\hat{\rho} \hat{\mathbf{u}} \frac{1}{2}|\hat{\mathbf{u}}|^{2}\right)+ \\
\frac{\gamma-1}{\gamma} M \hat{\nabla} \cdot(\hat{p} \hat{\mathbf{u}})=\frac{1}{R e P r} \hat{\nabla} \cdot(\hat{k} \hat{\nabla} \hat{T})+\frac{1}{R e} M^{2}(\gamma-1) \hat{\nabla} \cdot(\hat{\bar{\tau}} \hat{\mathbf{u}})
\end{array}\right.
$$

The working fluid being air, the Prandtl number is fixed, of order 1 . This leaves out two non-dimensional numbers to monitor wave propagation in the tube $(M$ and $R e)$. In order to characterize the streaming flow associated with a standing wave, the $\nabla$ operator should be separated into an axial and a radial part, that is a separate reference length scale for the radial direction has to be introduced, inducing that a supplementary non-dimensional number is needed. Menguy and Gilbert ${ }^{8}$ showed that this additional required number is the Shear number, defined as $S h=\frac{\delta_{\nu}}{R}, \delta_{\nu}=\sqrt{\frac{2 \nu}{\omega}}$ being the acoustic boundary layer thickness in the near wall region (with $\nu=\mu / \rho_{0}$ the kinematic viscosity).

Here, the acoustic wave amplitude ranges from linear $(M \ll 1)$ to weakly nonlinear. The Reynolds number compares inertial effects and viscous effects over the wavelength and is large with respect to 1 for the present range of audible frequencies. Then, the third dimensionless number compares the boundary layer thickness to the tube radius. The hypothesis $S h \ll 1$ corresponds to large tubes, which is the case in all the studies we deal with in this paper. Finally when working with air, the same flow behavior is expected for studies where $M \ll 1, R e \gg 1$ and $S h \ll 1$. This asymptotic similarity will be 
considered as valid for the numerical and experimental flows under study in the following. In order to be able to compare our experimental and numerical results to a reference, in the following section, two available models for streaming flow are briefly presented: a linear model developed by Bailliet et al. ${ }^{6}$ (used for quantitative comparison with direct numerical simulations and experiments in the slow streaming regime) and a nonlinear model developed by Menguy and Gilbert ${ }^{8}$ (used for the description of the evolution of the streaming flow for moderate streaming regime). The latter shows that when dealing with streaming flow, an additional dimensionless number has to be considered, as discussed in the following.

\section{B. Slow streaming}

In the literature, there are several analytical models for slow streaming ${ }^{2,3,7}$. In this paper, the model developed by Bailliet et $a l .{ }^{6}$ is used. This model is based on the fundamental equations presented above. Additional assumptions allow to obtain an expression of the second order time averaged axial velocity as function of the first order acoustic quantities. The streaming is supposed to be slow so that effect of inertia on the streaming motion is neglected by comparison with viscous effects. The solid is supposed to have an infinite heat capacity. Under these assumptions, the method of successive approximation is applied, the

first order quantities having a $e^{-i \omega t}$ dependence. Through time averaging a combination of the continuity equation and of the axial component of the Navier-Stokes equations written to the second order, the following equation is obtained:

$$
\frac{1}{R^{2}} \mu_{0} \nabla_{\eta}^{2}\left\langle u_{2}\right\rangle=\partial_{z} p_{2}+\partial_{z}\left(\rho_{0}\left\langle u_{1 z}^{2}\right\rangle\right)+\frac{1}{R} \nabla_{\eta}\left(\rho_{0}\left\langle u_{1 z} u_{1 \eta}\right\rangle\right)-\frac{1}{R^{2}} \nabla_{\eta}\left(\left\langle\mu_{1} \nabla_{\eta} u_{1 z}\right\rangle\right),
$$

where $\langle-\rangle$ denotes the time average and $\eta=\frac{r}{R}$, with $r$ the radial coordinate. An expression for the second order pressure gradient $\partial_{z} p_{2}$ is obtained by writing that, in a closed resonator, there is no net mass flow across the section, i.e. the second order time average mass flow rate across the cross section must be zero. Successive integration of the expression obtained yields the second order pressure gradient as a function of the first order acoustic quantities. In turn, successive integration of eq. (4) yields the axial streaming velocity (see formula (16) 
from Bailliet et $a .^{6}$ ). In the case of widely spaced parallel plates, this velocity is equal to the one obtained by RNW theory, but the calculation is valid also for narrow guides (that is in the near wall region of any guide) and it takes into account the dependence of viscosity on temperature. This calculation is also valid in the presence of a temperature gradient (not set in the present study). This analytical study will be used as a reference for our experimental and numerical results. However, high acoustic amplitudes will be considered here and therefore the slow streaming assumption is expected to be restrictive, and the effect of streaming inertia should be analyzed.

\section{Effect of inertia on streaming}

The only available theoretical study that is not restricted to slow streaming is the one by Menguy and Gilbert ${ }^{8}$. By writing the fundamental equations up to the third order in the main acoustic field followed by time averaging over the acoustic period, Menguy and Gilbert derived the governing equations for the streaming field in the boundary layer and in the main central part of the tube. Under the above presented assumptions $(M \ll 1, R e \gg 1$, $S h \ll 1$ ), an asymptotic analysis can be performed, allowing to derive the fluid behavior at the first and second order. A time averaging over the acoustic period yields equations for streaming flow both inside and outside the acoustic viscous/thermal boundary layer. Under the previous hypotheses on $M, R e$ and $S h$ numbers, streaming equations inside the boundary layer are always linear. Outside the boundary layer, streaming equations can be linear or nonlinear depending on the ratio between inertia and viscous terms. Menguy and Gilbert $^{8}$ showed that in the central part of the tube, the equation that governs the streaming velocity $\hat{u}_{s}$ has a unique nonlinear term

$$
\frac{S h^{2}}{M^{2}} \frac{1}{\hat{r}} \frac{\partial}{\partial \hat{r}}\left(\hat{r} \frac{\partial \hat{u}_{s}}{\partial \hat{r}}\right) \quad .
$$

This clearly shows that the outer streaming nonlinearity depends only upon one dimensionless number :

$$
R e_{N L}=\frac{M^{2}}{S h^{2}}
$$


called the nonlinear Reynolds number, that compares inertia and viscosity. For $R e_{N L}=O(1)$ the classical streaming models are no longer valid and the nonlinear effects of inertia must be taken into account. The latter cause distortion of the streaming velocity field in the tube and Menguy and Gilbert ${ }^{8}$ discuss the distortion of the toroidal vortex patterns. When $R e_{N L} \ll 1$, inertia terms are negligible with respect to viscous terms and the streaming equation reduces to the linear model of streaming (see Section II.B).

Finally, four dimensionless numbers are to be considered when dealing with Rayleigh streaming. At fixed $\operatorname{Pr}$, for asymptotic similarity when $M \ll 1, S h \ll 1$ and $R e \gg 1$, the nonlinear Reynolds number is expected to be the driving parameter for acoustic streaming. The behavior of the streaming flow will then be analyzed and discussed upon the values of the nonlinear Reynolds number.

\section{EXPERIMENTAL APPARATUS AND PROCEDURE}

The setup used to observe the phenomenon of acoustic streaming is shown in Fig. 1 and consists in a cylindrical tube connected at each end to a loudspeaker via connecting tubes designed to avoid separation effect related to the singularities in change of section. The cylindrical part of the waveguide has inner radius $R=19.5 \mathrm{~mm}$ and the waveguide is filled with atmospheric air. The total length of the wave guide is $L_{\exp }=2.13 \mathrm{~m}$ and the system is tuned on two distinct frequencies corresponding to two different resonant modes: the first mode corresponding to $L_{\exp }=\lambda / 2$ and the third mode corresponding to $L_{\exp }=3 \lambda / 2, \lambda$ being the wavelength of the fundamental mode.

In the following, results will be presented for acoustic streaming across one or two cells and whatever the working frequency, the position along the guide axis will be given by the axial coordinate $z$, with $z= \pm \lambda / 4$ corresponding to two adjacent streaming cells.

The acoustic wave is generated by two loudspeakers driven by a wave generator whose frequency and amplitude are controlled. Wood smoke is used as seeding particles in order to perform LDV measurements. The single component LDV system is a Dantec Dynamic Model 


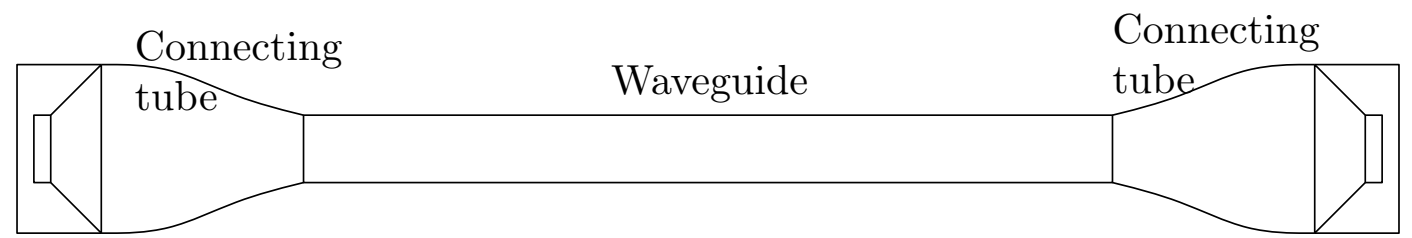

Loudspeaker

Loudspeaker

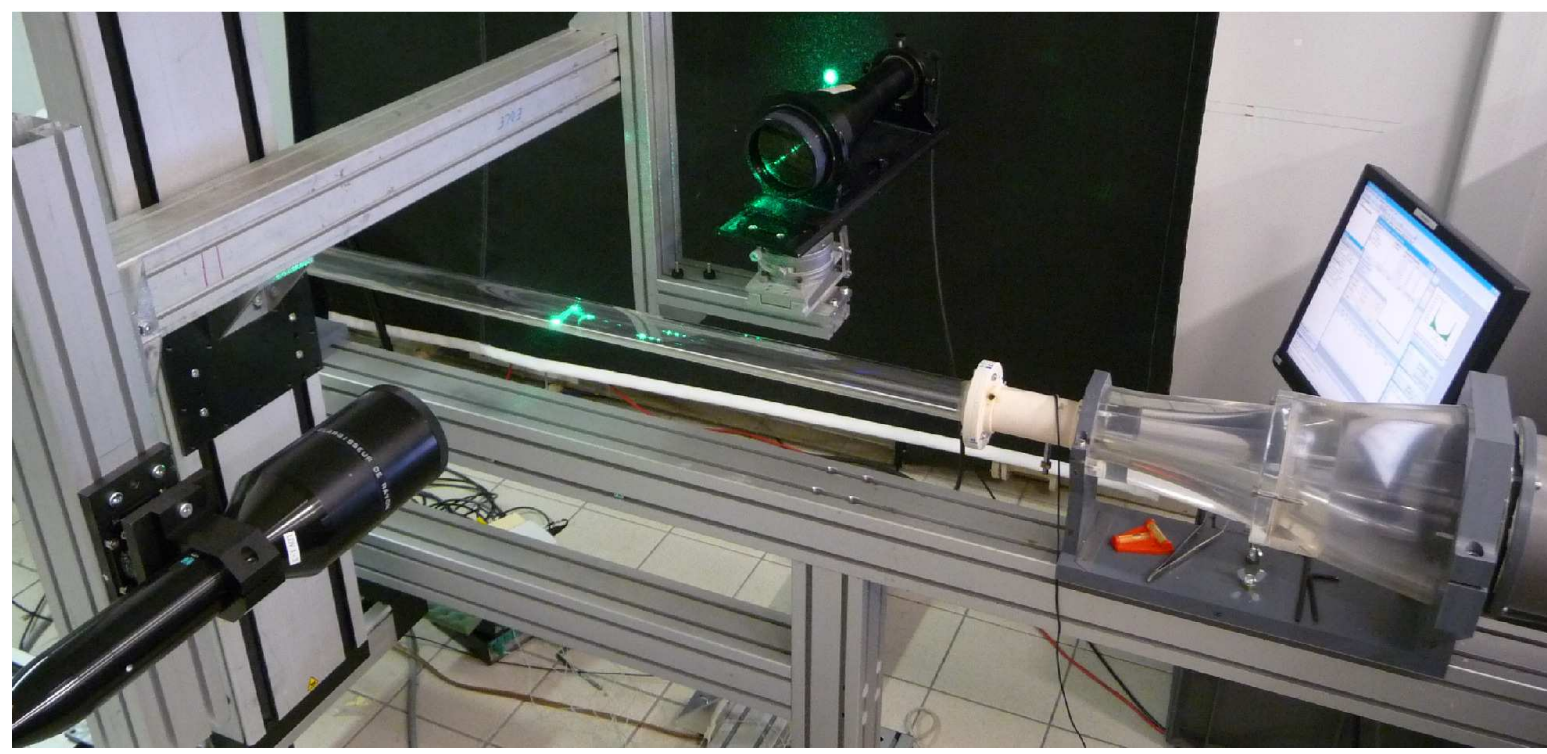

FIG. 1. (color online) Photo and diagram of the experimental apparatus.

2580, the probe is mounted on a three-axis positioning system. The argon krypton laser has an optical wavelength of $514.5 \mu \mathrm{m}$ and a power of $25 \mathrm{~W}$. The parameters of LDV system are adjusted for sound measurements ${ }^{20}$ and the axial streaming velocity is measured following the work of Valière et $a l^{21}$ along the centerline of the guide. The LDV measurements yield the Eulerian particle velocity. In agreement with Moreau et al. ${ }^{11}$, measurements were performed more than 26 min after the acoustic field is switched on and are stable after this time. Also, following this latter reference, in order to reach convergence in the measured streaming velocity, we choose to acquire either 70000 points or to stop acquisition after 10 seconds. This gives a good compromise between a sufficient number of measured tracer particles per point for a precise streaming velocity measurement and a small measuring time resulting from a correct seeding.

In order to get as close as possible to the isothermal boundary conditions used in the numerical calculation and because it was found in the literature that temperature gradients 
along the guide walls greatly influence Rayleigh streaming ${ }^{19}$, the resonator wall is forced to correspond to an isothermal boundary condition with the help of fans used to insure that the wall temperature is equal to the room temperature. The temperature is measured along one streaming cell using 7 thermocouples with a resolution of about $0.05^{\circ} \mathrm{C}$. These thermocouples are placed at the outer wall of the resonator and are insulated from the external environment by a polystyrene covering. Using fans to force heat transfer from the guide to the environment yields a residual temperature difference of $0.3^{\circ} \mathrm{C}$ between the acoustic velocity node and antinode at $R e_{N L}=31.8$. This temperature difference would be equal to $3.1^{\circ} \mathrm{C}$ if no fans were used.

\section{NUMERICAL APPROACH}

\section{A. Numerical method}

In order to initiate an acoustic standing wave in the cylindrical tube of length $L$, it is shaken in the longitudinal direction $z$, so that an harmonic velocity law is imposed, $\vec{V}(t)=(0, V(t))^{T}$, with $V(t)=z_{p} \omega \cos (\omega t), z_{p}$ being the amplitude of the tube displacement. The flow can be modeled by the compressible Navier-Stokes equations (1), expressed in the moving frame attached to the tube, so that a forcing source term $h^{s}$ is added to the system of equations (1). The model can be written in vector form :

$$
\frac{\partial w}{\partial t}+\frac{1}{r} \frac{\partial}{\partial r}\left[r\left(f-f^{v}\right)\right]+\frac{\partial}{\partial z}\left(g-g^{v}\right)=h+h^{s}
$$

where $w$ is the vector of conservative variables $(\rho, \rho u, \rho v, \rho E)^{T}, f$ and $g$ are the inviscid fluxes $f=\left(\rho u, \rho u^{2}+p, \rho u v, \rho E u+p u\right)^{T}$ and $g=\left(\rho v, \rho u v, \rho v^{2}+p, \rho E v+p v\right)^{T}, f^{v}$ and $g^{v}$ being

the viscous fluxes $f^{v}=\left(0, \tau_{r r}, \tau_{r z}, k \frac{\partial T}{\partial r}+u \tau_{r r}+v \tau_{r z}\right)^{T}, g^{v}=\left(0, \tau_{r z}, \tau_{z z}, k \frac{\partial T}{\partial z}+u \tau_{r z}+v \tau_{z z}\right)^{T}$. The source terms read $h=\left(0,-\frac{\tau_{\theta \theta}}{r}+\frac{p}{r}, 0,0\right), h^{s}=\left(0,0,-\rho \frac{d V}{d t},-\rho u \frac{d V}{d t}\right)^{T}$.

The model is numerically solved by using high order finite difference schemes, developed by Daru and Tenaud ${ }^{13}$. An upwind scheme, third order accurate in time and space, is used for convective terms, and a centered scheme, second order, is used for diffusion terms. 
Traveling shock waves are present in the flow for high acoustic levels, that generate numerical oscillations. However they are of weak intensity and produce very small oscillations that do not spoil the solution. Thus it was not necessary to add a costly shock capturing procedure.

The numerical procedure is briefly described thereafter. Denoting by $w_{i, j}^{n}$ the numerical solution at time $t=n \delta t$ and grid point $(r, z)=(i \delta r, j \delta z)$, with $\delta t$ and $\delta r, \delta z$ the time and space discretization steps respectively, the following Strang splitting procedure is used to obtain a second order accuracy every two time steps:

$$
w_{i, j}^{n+2}=L_{\delta r} L_{\delta z} L_{\delta z} L_{\delta r} w_{i j}^{n}
$$

where $L_{\delta r}\left(\operatorname{resp} . L_{\delta z}\right)$ is a discrete approximation of $L_{r}(w)=w+\delta t\left(-\frac{1}{r}(r f)_{r}+\frac{1}{r}\left(r f^{v}\right)_{r}+h+\right.$ $\left.h^{s}\right)\left(\right.$ resp. $\left.L_{z}(w)=w+\delta t\left(-g_{z}+g_{z}^{v}\right)\right)$. The $1 \mathrm{D}$ operators being similar in both directions, only the $r$ operator is described. The scheme is implemented as a correction to the second order Mac-Cormack scheme. It consists of three steps, as follows:

$$
\begin{aligned}
w_{i, j}^{*} & =w_{i, j}^{n}-\frac{\delta t}{r_{i, j} \delta r}\left[r_{i+\frac{1}{2}, j}\left(f_{i+1, j}-f_{i+\frac{1}{2}, j}^{v}\right)-r_{i-\frac{1}{2}, j}\left(f_{i, j}-f_{i-\frac{1}{2}, j}^{v}\right)\right]^{n}+\delta t\left(h+h^{s}\right)_{i, j}^{n} \\
w_{i, j}^{* *} & =w_{i, j}^{*}-\frac{\delta t}{r_{i, j} \delta r}\left[r_{i+\frac{1}{2}, j}\left(f_{i, j}-f_{i+\frac{1}{2}, j}^{v}\right)-r_{i-\frac{1}{2}, j}\left(f_{i-1, j}-f_{i-\frac{1}{2}, j}^{v}\right)\right]^{*} \\
w_{i, j}^{n+1} & =\frac{1}{2}\left(w_{i, j}^{n}+w_{i, j}^{* *}\right)+\frac{1}{r_{i, j}}\left(r_{i+\frac{1}{2}, j} C_{i+\frac{1}{2}, j}^{r}-r_{i-\frac{1}{2}, j} C_{i-\frac{1}{2}, j}^{r}\right)
\end{aligned}
$$

In $(7)$, the subscripts $\left(i \pm \frac{1}{2}, j\right)$ denote a value at the interface between cells $(i, j)$ and $(i \pm 1, j)$. The viscous fluxes are discretized at each interface using centered second order finite differences. The corrective terms $C_{i \pm \frac{1}{2}, j}^{r}$ provide the third order accuracy and upwinding for the inviscid terms. Details on the model can be found in Daru et al. ${ }^{14,15,22}$.

The physical boundary conditions employed in the moving frame are : no slip on the wall, symmetry at the ends of the tube, and isothermal walls.

The acoustic streaming is generated by the interaction of the imposed plane standing wave and the tube wall. Resonant conditions are imposed, for which $L=\lambda / 2, \lambda$ being the wavelength calculated for a dissipative gas. The boundary layer $\delta_{\nu}$ is of small thickness and must be correctly resolved by the discretization mesh. After several trials, it was found that a value of 5 points per boundary layer thickness is sufficient for reasonable accuracy of the simulations. All results presented below are thus obtained using a cartesian mesh of 
rectangular cells of constant size $\delta r$ and $\delta z$, composed of 500 points in the axial direction $z$, and of $5 \times R / \delta_{\nu}$ points in the radial direction.

The flow being assumed to be axisymmetric (at least in the range of parameters treated), only the region $0 \leq r \leq R$ and $0 \leq z \leq L$ was considered. Also, the scheme being fully explicit, the time step $\delta t$ is fixed such as to satisfy the stability condition of the scheme. In

all cases considered here, the time step limitation is acoustic, it reads $\delta t \leq \frac{1}{2} \delta r / c_{0}$. Taking $\delta t=\frac{1}{2} \delta r / c_{0}$ and $\delta r=\delta_{\nu} / 5$, this results in a number of time steps $N_{T}$ per period of oscillation proportional to $\sqrt{L}, N_{T}=1 /(f \delta t)=10 \sqrt{\frac{2 \pi c_{0}}{\nu}} \sqrt{L}$.

Because the LDV measurements yield the Eulerian particle velocity numerical results are based on the Eulerian time-average of fluid velocity. The numerical streaming velocity is obtained by calculating a simple mean value of the particle velocity over the discretized acoustic period, thus a time average made with respect to the fixed measuring position, for each mesh point.

\section{B. Simulation parameters}

A cylindrical tube is considered, initially filled with air at standard thermodynamic conditions, $p_{0}=101325 \mathrm{~Pa}, \rho_{0}=1.2 \mathrm{kgm}^{-3}, T_{0}=294.15 \mathrm{~K}$. The thermo-physical properties of air are $\mu_{0}=1.79510^{-5} \mathrm{kgm}^{-1} \mathrm{~s}^{-1}$ and $k_{0}=0.025 \mathrm{Wm}^{-1} \mathrm{~K}^{-1}$. Viscosity is expressed as a power law of temperature, $\mu(T)=\mu_{0}\left(T / T_{0}\right)^{0.77}$. Also for air, $\gamma=1.4$ and $r_{g}=287.06 \mathrm{Jkg}^{-1} \mathrm{~K}^{-1}$. The Prandtl number $\operatorname{Pr}$ is equal to 0.726. This results in an initial speed of sound $c_{0}=$ $343.82 \mathrm{~ms}^{-1}$.

In the experimental case presented in Section III, the half-wavelength is about $0.7 \mathrm{~m}$ long, which would yield a limiting time step corresponding to $N_{T} \approx 25000 \sqrt{L}$, that is 21000 iterations per period. Since transients of several hundreds of periods may be needed in order to reach stabilized streaming flow, several millions of iterations are necessary for each simulation. Considering these numerical constraints, a high-frequency wave is chosen for numerical calculations, with $f=20000 \mathrm{~Hz}$. Taking into account the viscous dissipation, 
this corresponds to a shorter wave guide with $L=8.396 \mathrm{~mm}$. The resulting boundary layer thickness is $\delta_{\nu}=1.5410^{-5} \mathrm{~m}$. For computational ease, the Shear number was fixed in the numerical simulations to be $1 / 40$.

The time step $\delta t=810^{-9} \mathrm{~s}$ is chosen in order to satisfy the numerical stability condition, corresponding to 6250 time iterations per period. The acoustic velocity produced in the wave guide depends on the imposed amplitude of the horizontal displacement $z_{p}$. It varies approximately linearly with $z_{p}$, for a given ratio $R / \delta_{\nu}$. Therefore it is possible via the only choice of $z_{p}$ to approximately set the value of the desired nonlinear Reynolds number $R e_{N L}$, so that to approximately fit the ones obtained in experimental configurations.

\section{RESULTS AND DISCUSSION}

As seen in sections III and IV several differences exist between experimental and simulated configurations. As discussed in section II these differences should not be associated with different behaviors of acoustic streaming if the nonlinear Reynolds number $R e_{N L}=\frac{M^{2}}{S h^{2}}$ is the same, and if the other relevant dimensionless numbers $S h=\frac{\delta_{\nu}}{R}, R e=\frac{c_{0}^{2}}{\nu \omega}$ and $M=\frac{U_{0}}{c_{0}}$ are of the same order of magnitude. The acoustic amplitude of the fundamental mode at the velocity antinode $z=0$ is chosen as the velocity reference $U_{0}$.

Table I summarizes then the values of these dimensionless parameters for both the experimental and numerical configurations together with the ones for several studies of the literature $8,10,12,19$ that are used for comparison with the present study.

As shown by Table I and as stated in the previous section IV, all numerical calculations were performed for a given frequency in air at atmospheric pressure, corresponding to one acoustic Reynolds number Re. Because the experimental setup could be tuned at two different resonant modes, two different Re numbers could be achieved. Whatever the frequency condition is in numerical and experimental configurations, the acoustic Reynolds number is always much larger than 1 . Therefore, we can expect similarity to hold between the different configurations studied here in terms of $R e$. 
When working on the $\lambda / 2$ experimental mode, the $S h$ number is $1.2 \cdot 10^{-2}$ (the corresponding experimental configurations are labeled $E_{1}$ and $E_{2}$ ), whereas when working on the $3 \lambda / 2$ experimental mode, the $S h$ number is $7.2 \cdot 10^{-3}$ (labeled $E_{3}$ to $E_{5}$ ). In the numerical simulations the $S h$ number is $2.5 \cdot 10^{-2}$. As will be shown in the following section, such differences in $S h$ numbers do not yield any significant difference in the acoustic streaming regime.

Finally, by changing the acoustic amplitude, the $M$ and $R e_{N L}$ numbers are modified. Since the $R e_{N L}$ number is the relevant parameter to study nonlinear acoustic streaming, numerical and experimental configurations were chosen such that the $R e_{N L}$ numbers are as close as possible for comparisons. The corresponding acoustic Mach number ranges from $8.2 \cdot 10^{-3}$ to $4 \cdot 10^{-2}$ for experiments and from $1.2 \cdot 10^{-2}$ to $1.5 \cdot 10^{-1}$ for numerical simulations, and therefore attention should be paid to the possible effects of such different dynamic ranges.

In the following Sections V.A-C, results for experiments and numerical simulations are presented for different ranges of $R e_{N L}$, then in Section V.D the effect of other dimensionless numbers are considered and discussed.

\section{A. Streaming velocity at low nonlinear Reynolds number}

The results presented in this section refer to situations where the nonlinear Reynolds number $R e_{N L}$ is smaller than 1 . The experimental case selected for this comparison at low nonlinear Reynolds number corresponds to $R e_{N L}=0.47\left(E_{1}\right)$, and the numerical simulation case corresponds to $R e_{N L}=0.25\left(N_{1}\right)$.

Figure 2 displays results from measurements and from numerical simulations for the axial streaming velocity component $u_{s}$ along the scaled central axis $z /(\lambda / 2)$ together with two different results from the analytical analysis presented in Section II corresponding to the numerical and experimental cases respectively. In all figures, the velocities are normalized by $U_{\text {Rayleigh }}=\frac{3}{8} \frac{U_{0}^{2}}{c_{0}}$ that corresponds to the amplitude of the streaming velocity in Rayleigh's theory. The nonlinear effects being negligible for these values of $R e_{N L}$, the axial streaming 


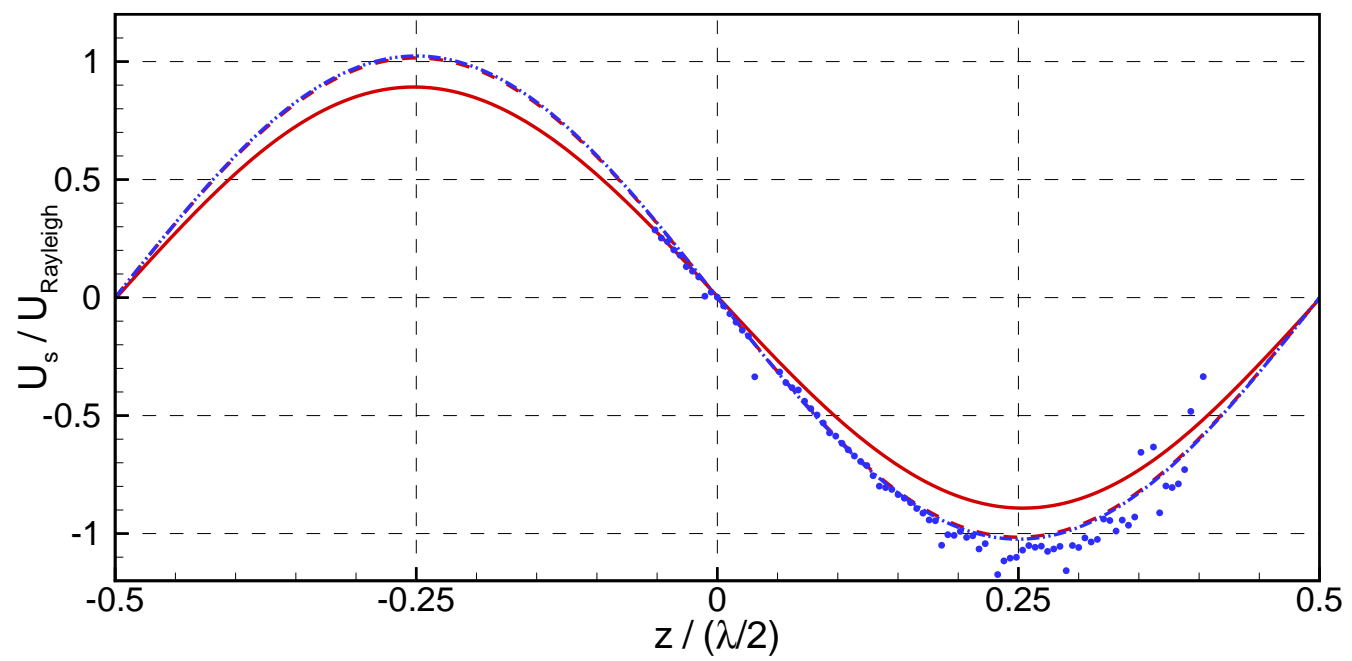

FIG. 2. (color online) Variation of the axial streaming velocity component along the central axis of the guide normalized by $U_{\text {Rayleigh }}=-\frac{3}{8} \frac{U_{0}^{2}}{c_{0}}$. (-) $N_{1}$ numerical simulation at $R e_{N L}=$ $0.25 ;(--)$ analytical results using the conditions of the simulation ; $\bullet$ ) $E_{1}$, experimental measurements $R e_{N L}=0.47$; (- - ) analytical results using the conditions of the experiment.

velocity should follow Rayleigh's law for linear streaming, that is $u_{\text {Rayleigh }}=-\frac{3}{8} \frac{U_{0}^{2}}{c_{0}} \sin \left(\frac{4 \pi z}{\lambda}\right)$ in the present coordinate system. Figure 2 shows that it is indeed the case for all results presented in the figure, except for a slight difference between numerical and both analytical and experimental results. The source of this difference is unclear. The acoustic wave is created in a different manner in the three studies (analytical, numerical and experimental). This can create discrepancies between the results. Moreover, it is important to recall that the numerical simulations are long and the streaming flow is of the second order. These are difficult conditions, especially for small values of the nonlinear Reynolds number. Therefore this difference in streaming velocity between numerical and analytical results is very satisfying.

The streamlines of the streaming flow are plotted in Fig. 3. Two outer streaming cells and two inner cells can be observed, corresponding to the classical Rayleigh streaming pattern. The streaming vortices are present either sides of the central axis and spaced at interval of $\lambda / 4$. For outer streaming, the flow along the central axis is directed toward the acoustic 
velocity antinodes and returns in the vicinity of the wall to complete a closed loop. In the near wall region, the inner streaming vortices have directions of rotation opposite to those of the outer cells. In the cylindrical resonator, those vortices form toroidal flows around the central axis.

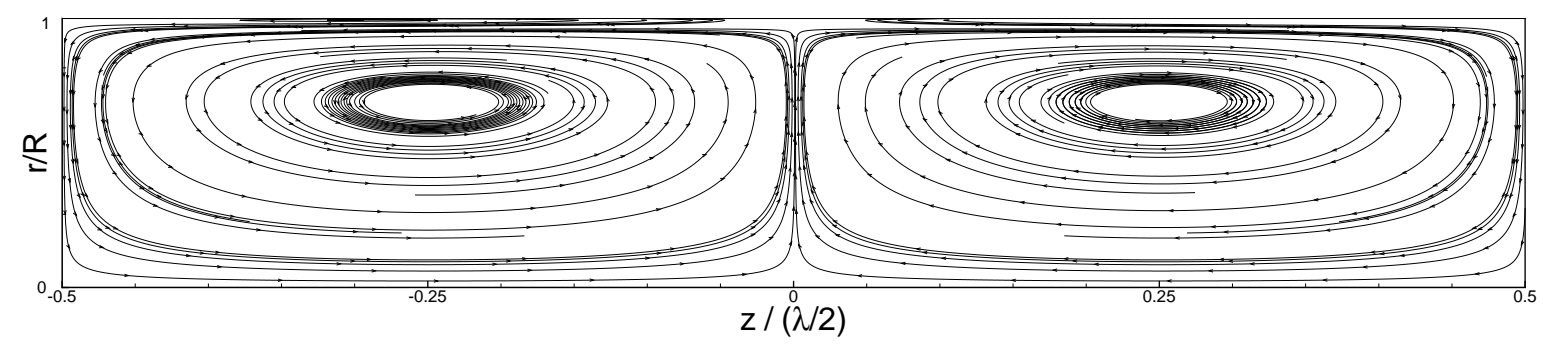

FIG. 3. Streamlines of the streaming flow, from numerical simulation at $R e_{N L}=0.25$.

Figure 4 shows the variation of the axial streaming velocity component along the radial axis on a cross-section positioned at $z=-\lambda / 8$, for the same cases as in Fig. 2. There is good agreement between all results in the core flow, away from the walls. Small discrepancies

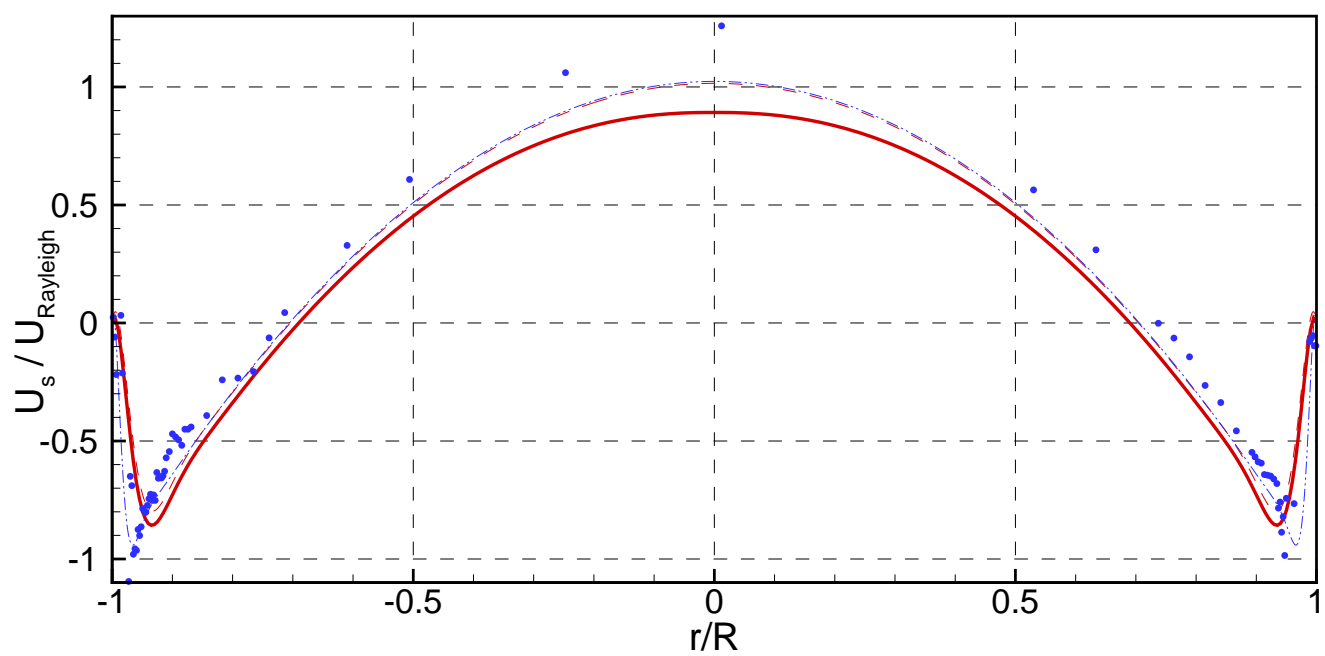

FIG. 4. (color online) Variation of the normalized axial streaming velocity component along the radial axis, at $z=-\lambda / 8$. (-) $N_{1}$, numerical simulation at $R e_{N L}=0.11$; (- -) analytical results using the conditions of the simulation ; $\bullet$ ) $E_{1}$, experimental measurements at $R e_{N L}=0.47 ;(-\cdot-)$ analytical results using the conditions of the experiment. 
between numerical and experimental results can be observed near the walls, when $0.8 R<r<$ $R$ that can be attributed to the difference between the Shear numbers in the experimental and numerical configurations. To cope with this effect of different Shear numbers, the evolution of the streaming radial dependency $u_{s}(r)$ will no further be discussed, but only the evolution of the axial dependency $u_{s}(z)$ along the axis with $R e_{N L}$.

Also, given the similarity between the two analytical curves along the center line, it is chosen in the following to plot only the analytical result corresponding to the experimental configuration.

\section{B. Streaming velocity at moderate nonlinear Reynolds number}

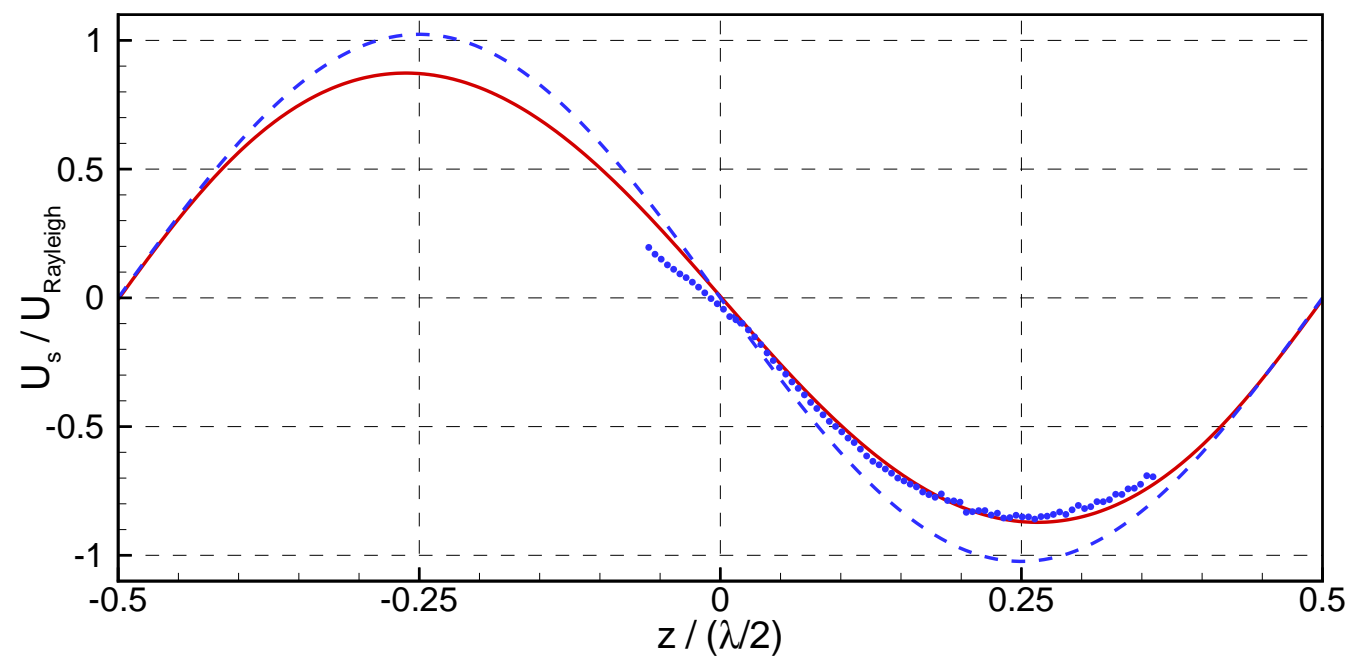

FIG. 5. (color online) Variation of the normalized axial streaming velocity component along the central axis of the guide. (- -) Analytical results using the conditions of the experiment ; (•) $E_{2}$, experimental measurements at $R e_{N L}=4.8 ;(-) N_{2}$, numerical simulation $R e_{N L}=$ 4.91.

Figures 5, 6, 7 display results of measurements and calculations for the centerline streaming velocity along the axis for increasing values of $R e_{N L}$, up to $R e_{N L} \approx 15$. They show that as $R e_{N L}$ increases, the streaming velocity departs from the linear theoretical expectation, 


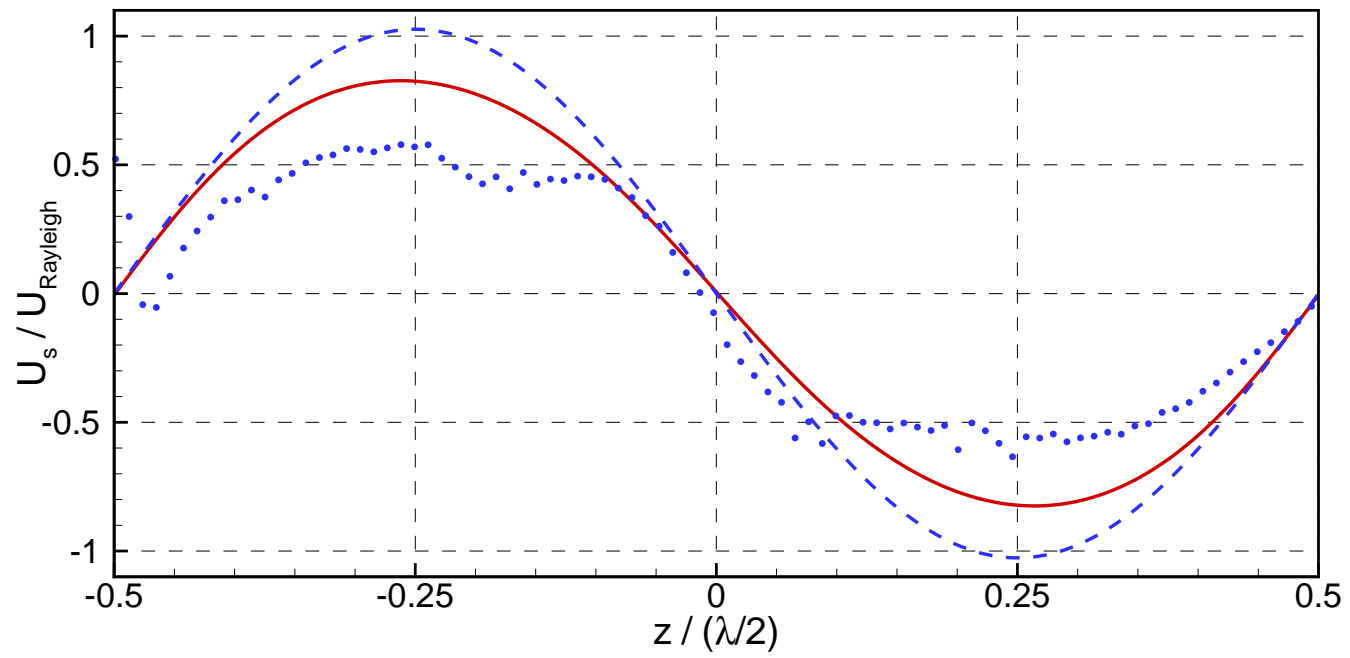

FIG. 6. (color online) Variation of the normalized axial streaming velocity component along the central axis of the guide. (- -) Analytical results using the conditions of the experiment ; $(\bullet) E_{3}$, experimental measurements at $R e_{N L}=7.7 ;(\boldsymbol{-}) N_{3}$, numerical simulation at $R e_{N L}=7.93$.

especially at streaming antinodes (i.e. $z /(\lambda / 2)= \pm 0.25$ in the considered figures) where it gets significantly lower than Rayleigh expectation. As shown in Fig. 6, the streaming structure keeps its symmetry with respect to the $z=0$ position, both in numerical simulations and in experimental measurements. It was checked that this symmetry is kept even for higher amplitude, and therefore, in the rest of the figures, measurements are presented along one cell only (from about $z=0$ to $z / \lambda / 2=0.5$ ).

The figures clearly show a continuous decrease of the maximum value of the streaming velocity as $R e_{N L}$ increases both in the experimental results and the numerical simulations even so the decrease is not as pronounced in the numerical simulations as in experiments (see Figure 7). Potential sources of this discrepancy will be discussed in Section V.D. 


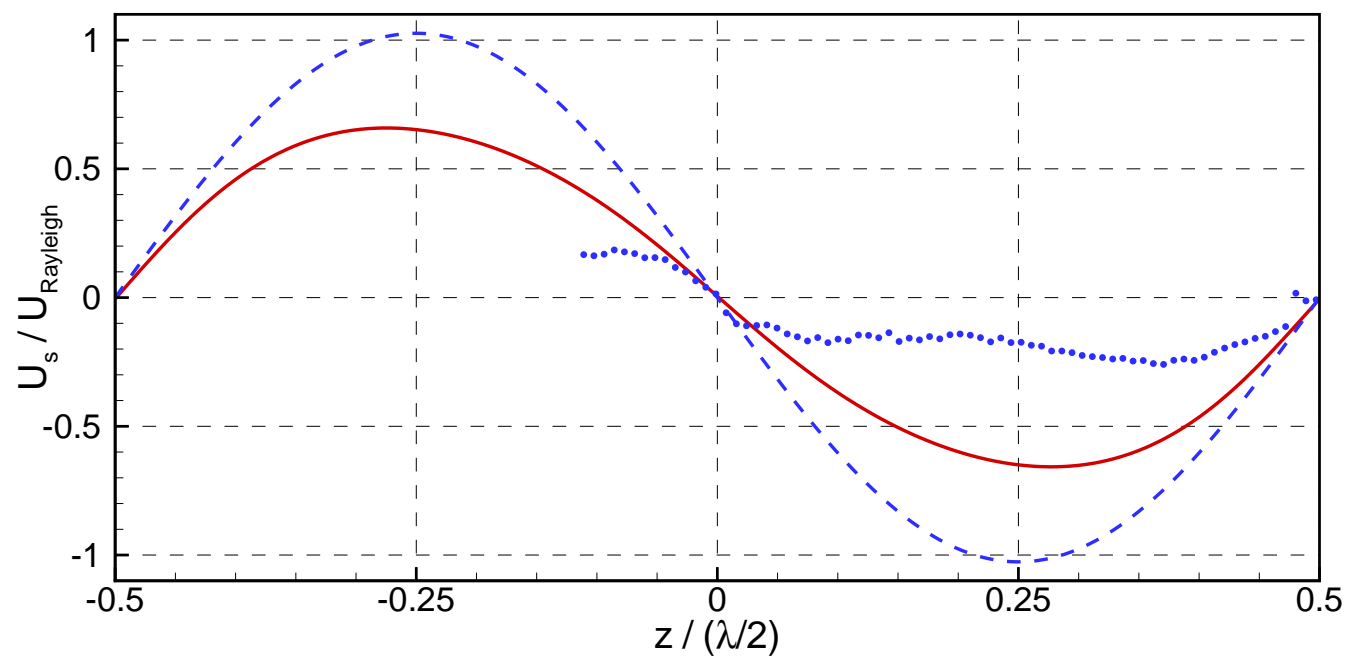

FIG. 7. (color online) Variation of the normalized axial streaming velocity component along the central axis of the guide. (- -) Analytical results using the conditions of the experiment ; $(\bullet) E_{4}$, experimental measurements at $R e_{N L}=14.6 ;(-) N_{4}$, numerical simulation at $R e_{N L}=15.2$.

\section{Streaming velocity at high nonlinear Reynolds number}

As the Reynolds number is further increased, as shown in Fig. 8, the difference between measured and calculated streaming on one hand, and linear theory on the other hand is even more pronounced.

In Figure 8, experimental results are very different from the linear theory along the axis except near the streaming velocity nodes $(z=0$ and $z /(\lambda / 2)=0.5$ positions). In the $z=0$ region, the curve for the measured streaming velocity along the central axis has successive changes of slope. The maximum measured streaming velocity keeps on decreasing when $R e_{N L}$ is increased until it crosses the zero-velocity axis for $R e_{N L}>30$, corresponding to the apparition of a new streaming cell.

The numerical simulation shows an overall similar behavior, although the new cells appearing around the central axis are smaller in the $z$ extent. Near the ends of the guide small additional cells appear in the numerical simulations. The streamlines obtained numerically 


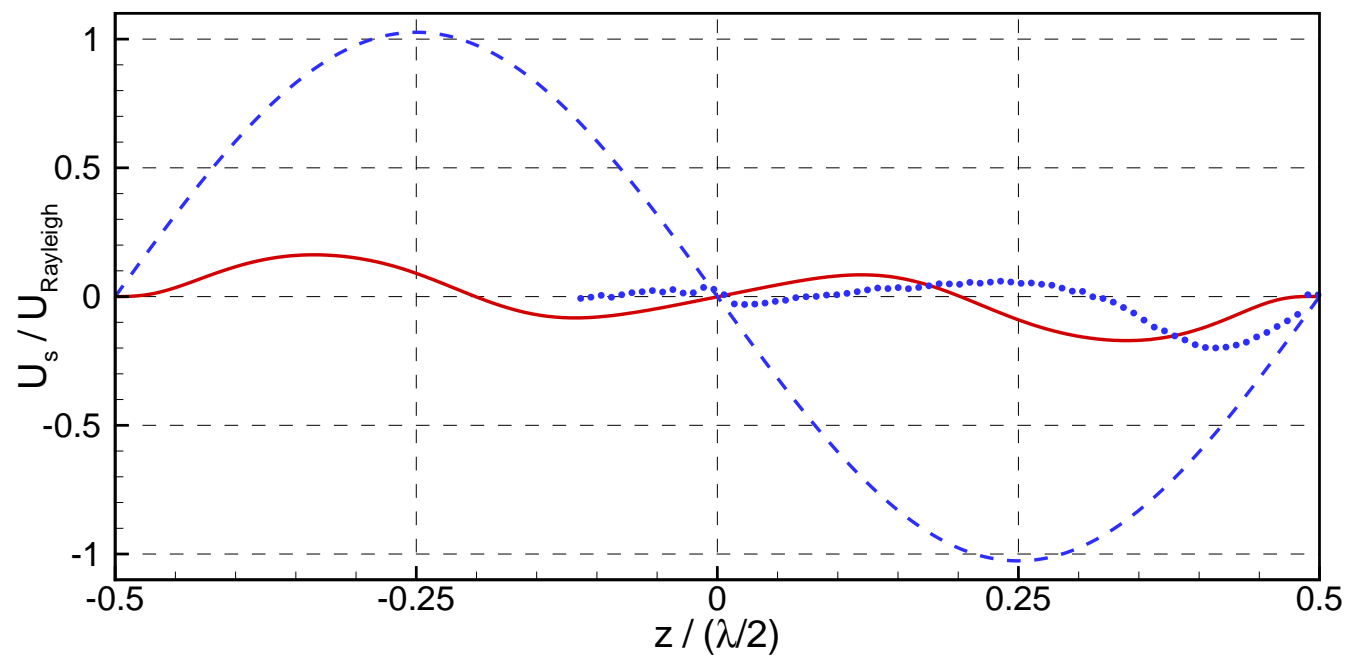

FIG. 8. (color online) Variation of the normalized axial streaming velocity component along the central axis of the guide. (- -) Analytical results using the conditions of the experiment ; $(\bullet) E_{5}$, experimental measurements at $R e_{N L}=31.8 ;(-) N_{5}$, numerical simulation at $R e_{N L}=38.6$.

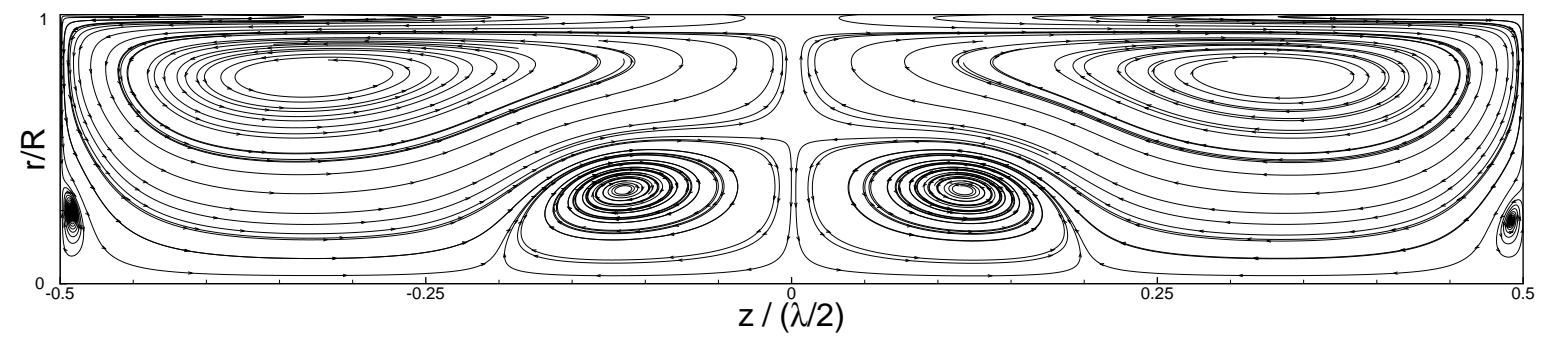

FIG. 9. Streamlines of the streaming flow, from numerical simulation at $R e_{N L}=38.6$.

are shown on Figure 9 enabling the visualization of the new streaming cells. Even if the experimental and numerical results do not superimpose perfectly, it is remarkable that the same qualitative and overall quantitative behaviors are observed for close values of the $R e_{N L}$ number. Figure 10 shows the numerical results for the modification of the streaming field as $R e_{N L}$ increases, clearly exhibiting the emergence of new streaming cells for $R e_{N L}$ greater than 30 . 


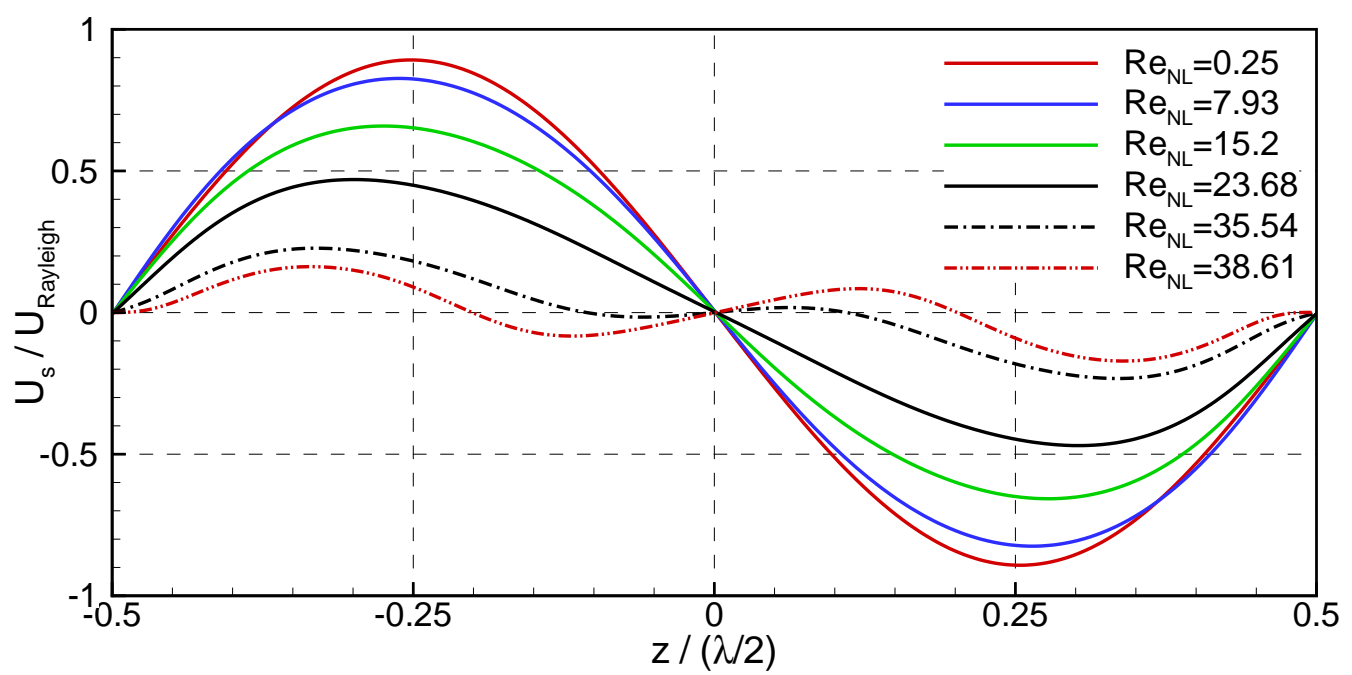

FIG. 10. (color online) Numerical simulations: variation of the normalized axial streaming velocity component along the central axis of the guide for several values of $R e_{N L}$.

\section{Discussion}

As seen in the previous sections, numerical, experimental and analytical results all agree for small values of $R e_{N L}$. As $R e_{N L}$ increases, the tendencies are similar for numerical simulations and experiments: the levels of dimensionless streaming decrease and new streaming cells appear at a critical value $R e_{N L} \simeq 30$. In order to identify a physical source for this evolution of streaming with $R e_{N L}$, several hypotheses are discussed in the following sections on the basis of the present literature review.

\section{Thermal effects}

Following Thompson et al. ${ }^{19}$, the influence of thermal effects can be questioned. They performed measurements in a standing wave guide for nonlinear Reynolds numbers up to 22, the three other dimensionless numbers $(M, R e$ and $S h)$ respecting the above mentioned asymptotic conditions of our study, see Table I. They explored the evolution of the streaming for three different thermal conditions : isothermal, uncontrolled and insulated and for different nonlinear Reynolds numbers. They observed that when the magnitude of the tem- 
perature gradient was increased by changing the thermal condition for a given high nonlinear Reynolds number, the magnitude of the streaming decreased and the shape of the streaming cell became increasingly distorted. They found that the thermoacoustically induced axial temperature gradient strongly influences the axial component of the acoustic streaming velocity and concluded that the deviation from the theories of Rott (characterizing slow streaming with an axial temperature gradient) is due to the influence of thermal effect more than fluid inertia.

In our numerical simulations, isothermal boundary conditions are imposed and thermal effects are accounted for. Nevertheless, the axial streaming velocity profile is deformed with increasing $R e_{N L}$. The same tendency is observed in the experimental results presented in Fig. 5-8, even if the thermal condition maintained for our set up is only quasi-isothermal (as a $0.3^{\circ} \mathrm{C}$ temperature difference between acoustic velocity node and acoustic velocity antinode is measured). For $R e_{N L}=31.8$ the streaming flow already presents additional vortices compared to Rayleigh expectation, as shown by the change of sign of the axial streaming velocity in Fig. 8.

Therefore, if we agree that thermal effects are very important in understanding acoustic streaming (the difference between experimental and numerical thermal conditions could be at the origin of the difference in results) it appears from our study that the presence of a temperature gradient is not the cause of the divergence from slow streaming expectation at high $R e_{N L}$ observed both numerically and experimentally.

\section{Irregular streaming}

Another possible cause of streaming distortion at high amplitudes exposed in the numerical and experimental literature is the change of regime from "regular" (or classical) to "irregular" streaming. In the literature the term "regular" is used when streaming flow exhibits the well-known patterns (toroidal outer and inner cells), while the term "irregular" is used to characterize any streaming flow that deviates from these patterns. 
Aktas and Farouk ${ }^{10}$ studied acoustic streaming in a 2D rectangular channel numerically. They found that there is a limit in the value of the enclosure height to wavelength ratio above which the streaming flow structures are always irregular and complex. Since their working frequency is constant, it is equivalent to consider a limit value of the inverse of the Shear number. Based on their numerical results, they estimate this limit value of $1 / S h$ between 30 and 40. In the present paper, results for values greater or equal to 40 are shown, and we do observe regular streaming for low values of $R e_{N L}$ (cases $E_{1}$ and $N_{1}$ ). The results presented by Aktas and Farouk ${ }^{10}$ do not exhibit any clear correlation between the regularity of the streaming and the value of $R e_{N L}$. For example, for two nonlinear Reynolds numbers corresponding to slow streaming cases $R e_{N L}=0.10$ (called $C$-3 in their paper) and $R e_{N L}=0.08(D-2)$, they observe two different streaming structures, respectively classical and irregular. The case with irregular streaming structure has a value of $1 / S h$ of 40. This last case is almost similar to our simulation $N_{1}$ where $R e_{N L}=0.25$, shown in Fig. 3. In this figure, the streaming velocity field is clearly regular. Although our results are obtained for axisymmetrical configurations, whereas those of Aktas and Farouk ${ }^{10}$ concern a rectangular enclosure, previous work ${ }^{14}$ showed similar behavior in planar geometry compared to the present observations in the axisymmetrical case. This is indeed coherent with the asymptotic approach.

In the experimental literature, Nabavi et al. ${ }^{12}$ mentioned irregular Rayleigh streaming for nonlinear Reynolds number above 20. Their experiments were performed in the case of isothermal boundary conditions. They found that for nonlinear Reynolds number above 25, the classical streaming deforms to an irregular and complex flow structure. The deformation they observe is similar to what we observe for some of their irregular cases, however the pattern of the irregular streaming is not always consistent amongst the experiments and with the value of $R e_{N L}$. In the above presented results, the streaming flow remains stable, consistent and show a continuous evolution from low to high amplitudes. Measurements have been repeated several times, they were always performed for steady flow and the results obtained are very reproducible. 
Finally, we choose not to use the terms "regular" or "irregular" to describe the streaming flow regimes observed both numerically and experimentally. Instead, we would rather describe them as "regular" streaming flow regimes with either two $\left(R e_{N L}<30\right)$ or three $\left(R e_{N L}>30\right)$ toroidal structures, with a smooth transition as $R e_{N L}$ increases.

\section{Nonlinear propagation}
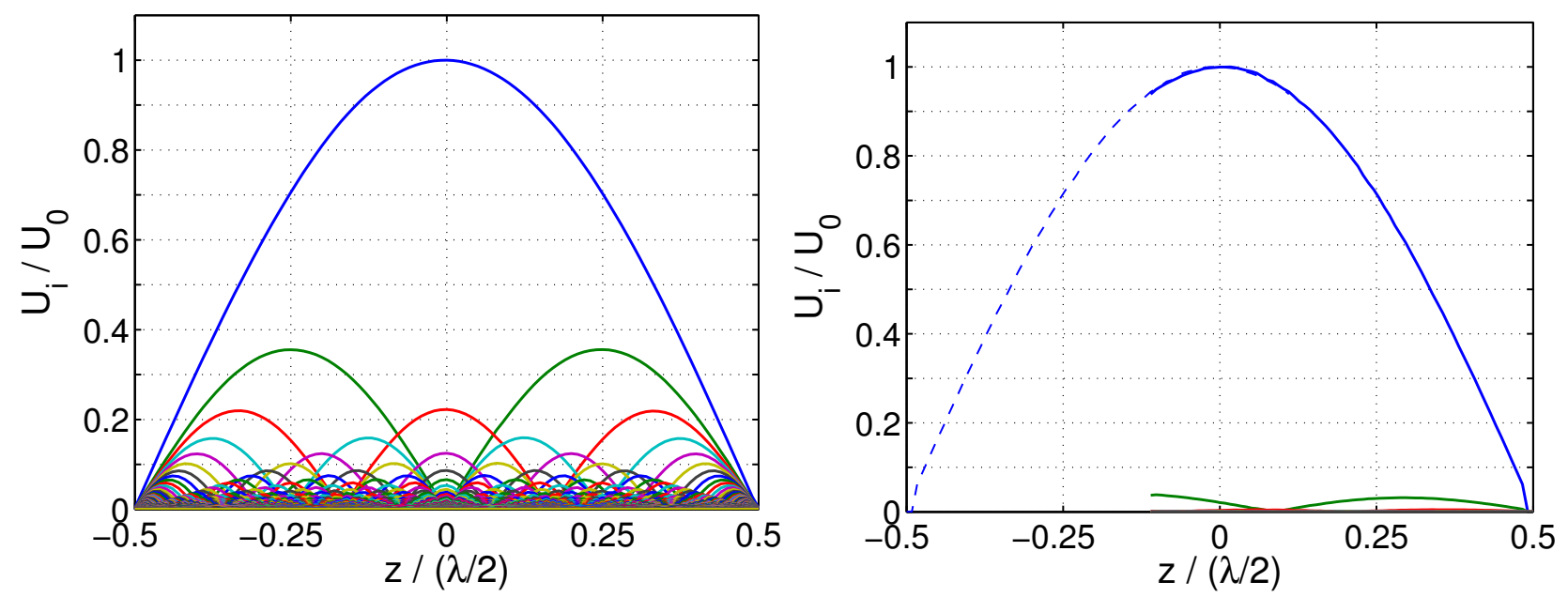

FIG. 11. (color online) Distribution of the harmonics along the guide. (a) Numerical simulation $N_{5}$; (b) Experimental study $E_{5}$.

Our analysis has been done under the assumption of low Mach number. But as the nonlinear Reynolds number increases, so does the Mach number, creating situations where nonlinear acoustic propagation should be of importance, thus the generation of higher harmonics has to be considered. This brings up the question of higher harmonics influence on the streaming in competition with inertial effects associated with high values of nonlinear Reynolds number.

Figure 11 shows the harmonic distribution of the acoustic field, obtained by projecting the total velocity signals on a sine basis, in both numerical (Fig.11.a) and experimental 
(Fig.11.b) results for $R e_{N L} \approx 30$. It shows that the nonlinear propagation induces the existence of shockwaves in the simulations whereas the magnitude of higher harmonics never reaches more than $4 \%$ of the magnitude of the fundamental in the experiments, due to the difference in geometrical configurations between experimental and numerical studies. Now for a first approach a superposition principle is applied in order to identify the acoustic streaming due to multiple frequency components. But this crude assumption cannot cope with nonlinear effects. So, as stated in section II.C, because the boundary layer can be considered as a region where the streaming is linear, we choose to superimpose the streaming fields resulting from higher harmonics in the vicinity of this region. We choose the particular radial position corresponding to the limit between inner and outer streaming cell, that is at $(R-r) \approx 3 \delta_{\nu}$, at which the streaming is expected to be close to linear streaming. Figure 12 shows the streaming velocity obtained from the numerical simulation at $R e_{N L}=38.6\left(N_{5}\right)$, the analytical calculation resulting from the fundamental component of the numerical signal, and the analytical calculation resulting from the linear superposition of all the harmonic components of the numerical signal. First, the distortion of the streaming close to the boundary layer (Fig. 12) is found to be much weaker than in the core of the resonator (Fig. 8 and Fig. 10). The effect of the harmonics superposition at $(R-r) \approx 3 \delta_{\nu}$ results in a shift of the streaming velocity maxima towards the acoustic velocity nodes associated with a small change in the velocity magnitude. The same type of distortion can be observed on the numerical profile (Fig. 12), but with a stronger change in the velocity magnitude (the maximum value and the slope of the curve at $z=0$ are much smaller). Moreover, as the distortion in acoustic streaming is qualitatively the same in both experimental and numerical study along the centerline of the guide, we conclude that while the harmonic content of experimental and numerical signals are very different, the influence of higher harmonics is not the main cause of the divergence of the streaming field from Rayleigh's expectation.

Finally we are left with only one hypothesis to explain this divergence: the inertial effects cannot be neglected anymore when the streaming is fast. 


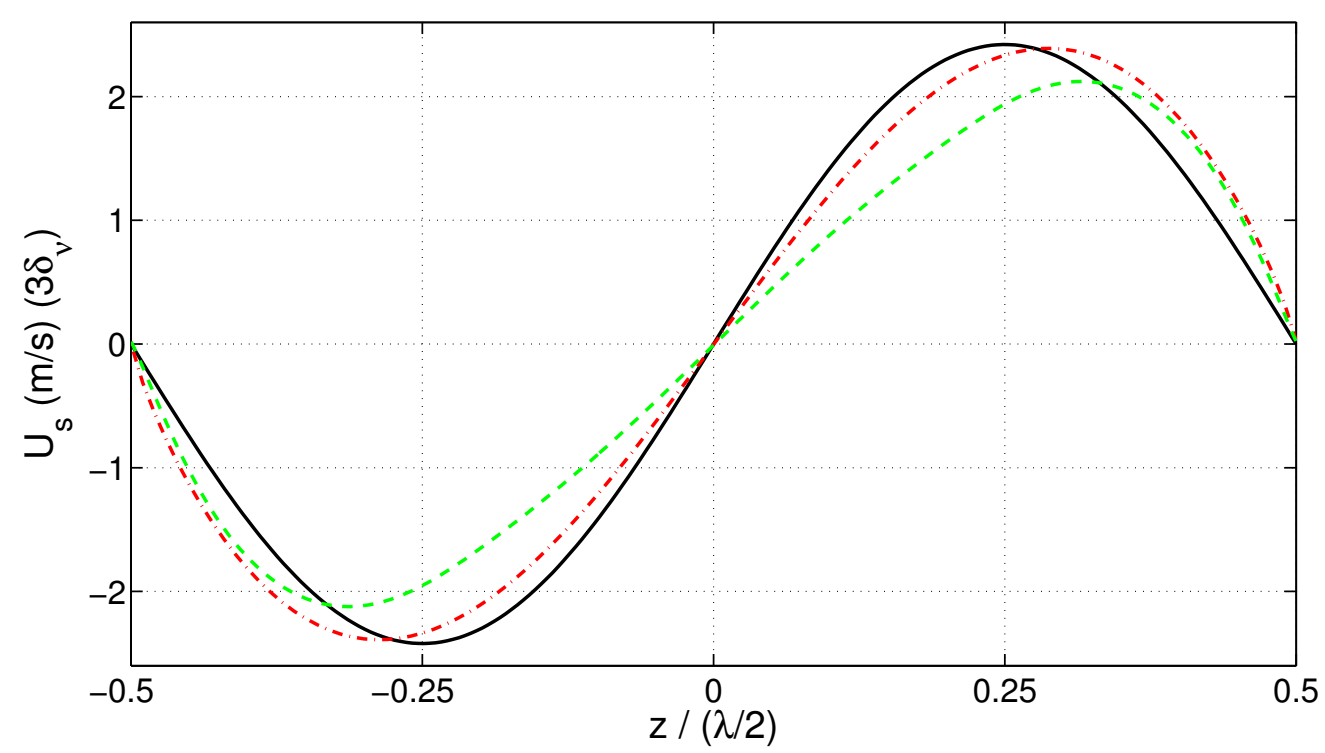

FIG. 12. (color online) Streaming velocity profile along the axis of the guide at $(R-r) \approx$ $3 \delta_{\nu}$, for $R e_{N L}=38.6$. (-) Analytical solution ; (--) Streaming velocity from analytical superposition of frequency components of the numerically calculated acoustic velocity; (- -) Numerical simulation.

\section{CONCLUSION}

In this paper a coupled experimental and numerical study of Rayleigh streaming from low to high acoustic amplitudes was conducted. As expected, slow streaming (both numerically and experimentally obtained) agrees with analytical slow streaming expectations for small $R e_{N L}$. The divergence from these analytical results that occurs when the $R e_{N L}$ is increased is very similar for numerical and experimental results. As $R e_{N L}$ increases, the cell centers are pushed towards the acoustic velocity nodes and the maximum axial streaming velocity decreases with respect to the analytical Rayleigh reference amplitude. It was also shown that for $R e_{N L}$ larger than about 30, additional outer cells are formed through a smooth transition between coherent streaming flow patterns. This is why the streaming flow regimes can be called "regular" throughout the transition between two $\left(R e_{N L}<30\right)$ and three $\left(R e_{N L}>30\right)$ toroidal structures. After having considered all potential sources for the observed divergence from slow streaming flow, we have concluded that inertial effects on acoustic streaming are 
the main source. Logically the $R e_{N L}$ is the driving parameter for the acoustic streaming in the regime considered here.

\section{Acknowledgments}

All the experiments were conducted at the Pprime Institute. The authors wish to aknowledge the technical support of Pascal Biais, Laurent Philippon and Philippe Szeger. 


\section{References}

1 Rayleigh, L. (1884). "On the circulation of air observed in Kundt's tubes, and on some allied acoustical problems", Philos. Trans. R. Soc. London 175, 1-21.

2 Nyborg, W. L. (1965). "Acoustic streaming", in Physical Acoustics, edited by W. P. Mason (Academic, New York, 1965), Vol. 2B, Chap. 11, 290-295.

3 Westervelt, P. J. (1953). "The theory of steady rotational flow generated by a sound field", J. Acoust. Soc. Am. 25, 60-67.

4 Olson, J. and Swift, G. (1997). "Acoustic streaming in pulse tube refrigerators: tapered pulse tubes", Cryogenics 37, $769-776$.

${ }^{5}$ Hamilton, M. F., Ilinskii, Y. A., and Zabolotskaya, E. A. (2003). "Thermal effects on acoustic streaming in standing waves", J. Acoust. Soc. Am. 114, 3092-3101.

6 Bailliet, H., Gusev, V., Raspet, R., and Hiller, R. A. (2001). "Acoustic streaming in closed thermoacoustic devices", J. Acoust. Soc. Am. 110, 1808-1821.

7 Hamilton, M. F., Ilinskii, Y. A., and Zabolotskaya, E. A. (2003). "Acoustic streaming generated by standing waves in two-dimensional channels of arbitrary width", J. Acoust. Soc. Am. 113, 153-160.

8 Menguy, L. and Gilbert, J. (1999). "Nonlinear effect of the inertia of the fluid on acoustic streaming in cylindrical guides", J. Acoust. Soc. Am. 105, 958-958.

9 Marx, D. and Blanc-Benon, P. (2004). "Computation of the mean velocity field above a stack plate in a thermoacoustic refrigerator", Comptes Rendus Mécanique 332, 867 874.

10 Aktas, M. K. and Farouk, B. (2004). "Numerical simulation of acoustic streaming generated by finite-amplitude resonant oscillations in an enclosure", J. Acoust. Soc. Am. 116, $2822-2831$.

11 Moreau, S., Bailliet, H., and Valière, J.-C. (2008). "Measurements of inner and outer streaming vortices in a standing waveguide using laser doppler velocimetry", J. Acoust. Soc. Am. 123, 640-647. 
12 Nabavi, M., Siddiqui, K., and Dargahi, J. (2009). "Analysis of regular and irregular acoustic streaming patterns in a rectangular enclosure", Wave Motion 46, 312 - 322 .

13 Daru, V. and Tenaud, C. (2004). "High order one-step monotonicity-preserving schemes for unsteady compressible flow calculations", J. Comp. Phys. 193, 563 - 594.

14 Daru, V., Baltean-Carlès, D., Weisman, C., Debesse, P., and Gandikota, G. (2013). "Two-dimensional numerical simulations of nonlinear acoustic streaming in standing waves", Wave Motion 50(5), 955 - 963.

15 Daru, V. and Gloerfelt, X. (2007). "Aeroacoustic computations using a high order shockcapturing scheme", AIAA J. 45, 2474-2486.

16 Sharpe, J., Greated, C., Gray, C., and Campbell, D. M. (1989). "The measurements of acoustic streaming using particle image velocimetry", Acustica 68, 168 - 172.

17 Campbell, M., Cosgrove, J., Greated, C., Jack, S., and Rockliff, D. (2000). "Review of LDA and PIV applied to the measurement of sound and acoustic streaming", Optics and Laser Technology 32, 629 - 639, optical methods in heat and fluid flow.

18 Thompson, M. and Atchley, A. (2005). "Simultaneous measurement of acoustic and streaming velocities in a standing wave using laser doppler anemometry", J. Acoust. Soc. Am. 117, 1828-1838.

19 Thompson, M., Atchley, A., and Maccarone, M. (2005). "Influences of a temperature gradient and fluid inertia on acoustic streaming in a standing wave", J. Acoust. Soc. Am. 117, 1839-1849.

20 Moreau, S., Bailliet, H., Valière, J.-C., Boucheron, R., and Poignand, G. (2009). "Development of laser techniques for acoustic boundary layer measurements. Part II: Comparison of LDV and PIV measurements to analytical calculation", Acta Acustica united with Acustica 95, 805-813.

21 Valière, J.-C., Moreau, S., and Bailliet, H. (2009). "Development of laser techniques for acoustic boundary layer measurements. Part I: LDV signal processing for high acoustic displacements", Acta Acustica united with Acustica 95, 585-594.

22 Daru, V. and Tenaud, C. (2001). "Evaluation of TVD high resolution schemes for un- 
steady viscous shocked flows", Computers and Fluids 30, 89 - 113. 
TABLE I. Non-dimensional numbers used for the present study together with those of several studies in the literature.

\begin{tabular}{|c|c|c|c|c|c|}
\hline Reference & Name & $R e_{N L}$ & $M$ & $S h$ & $R e$ \\
\hline & $E_{1}$ & 0.47 & $8.2 \cdot 10^{-3}$ & \multirow{2}{*}{$1.2 \cdot 10^{-2}$} & \multirow{2}{*}{$1.4 \cdot 10^{7}$} \\
\hline & $E_{2}$ & 4.8 & $2.6 \cdot 10^{-2}$ & & \\
\hline Experiments & $E_{3}$ & 7.7 & $2 \cdot 10^{-2}$ & \multirow{3}{*}{$7.2 \cdot 10^{-3}$} & \multirow{3}{*}{$5.2 \cdot 10^{6}$} \\
\hline \multirow[t]{4}{*}{ (present study) } & $E_{4}$ & 14.6 & $2.7 \cdot 10^{-2}$ & & \\
\hline & $E_{5}$ & 31.8 & $4 \cdot 10^{-2}$ & & \\
\hline & $N_{1}$ & 0.25 & $1.2 \cdot 10^{-2}$ & \multirow{5}{*}{$2.5 \cdot 10^{-2}$} & \multirow{5}{*}{$6.3 \cdot 10^{4}$} \\
\hline & $N_{2}$ & 4.91 & $5.5 \cdot 10^{-2}$ & & \\
\hline \multirow{3}{*}{$\begin{array}{l}\text { Numerical } \\
\text { (present study) }\end{array}$} & $N_{3}$ & 7.93 & $7.0 \cdot 10^{-2}$ & & \\
\hline & $N_{4}$ & 15.2 & $9.7 \cdot 10^{-2}$ & & \\
\hline & $N_{5}$ & 38.6 & $15 \cdot 10^{-2}$ & & \\
\hline Menguy \& Gilbert ${ }^{8}$ & & {$[0.5-2]$} & $<<1$ & $<<1$ & $>>1$ \\
\hline Thompson et al. ${ }^{19}$ & & {$[2.1-22]$} & {$[8-25] \cdot 10^{-3}$} & $5.3 \cdot 10^{-3}$ & $4.1 \cdot 10^{6}$ \\
\hline Nabavi et al. ${ }^{12}$ & & {$[6.6-78]$} & {$[7.7-34] \cdot 10^{-3}$} & {$[3-4] \cdot 10^{-3}$} & {$[1-1.9] \cdot 10^{6}$} \\
\hline Aktas \& Farouk ${ }^{10}$ & & {$[0.035-16.4]$} & {$[3.7-79] \cdot 10^{-3}$} & {$[2-10] \cdot 10^{-2}$} & $6.6 \cdot 10^{4}$ \\
\hline
\end{tabular}




\section{List of Figures}

FIG. 1 (color online) Photo and diagram of the experimental apparatus. . . . . . 12

FIG. 2 (color online) Variation of the axial streaming velocity component along the central axis of the guide normalized by $U_{\text {Rayleigh }}=-\frac{3}{8} \frac{U_{0}^{2}}{c_{0}}$. (-) $N_{1}$ numerical simulation at $R e_{N L}=0.25 ;(--)$ analytical results using the conditions of the simulation ; $(\bullet) E_{1}$, experimental measurements $R e_{N L}=0.47 ;(-\cdot)$ analytical results using the conditions of the experiment. . . . . . . . . 18

FIG. 3 Streamlines of the streaming flow, from numerical simulation at $R e_{N L}=0.25 . \quad 19$

FIG. 4 (color online) Variation of the normalized axial streaming velocity component along the radial axis, at $z=-\lambda / 8$. (-) $N_{1}$, numerical simulation at $R e_{N L}=$ $0.11 ;(--)$ analytical results using the conditions of the simulation ; $\bullet$ ( $E_{1}$, experimental measurements at $R e_{N L}=0.47 ;(-\cdots)$ analytical results using

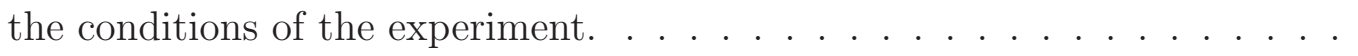

FIG. 5 (color online) Variation of the normalized axial streaming velocity component along the central axis of the guide. (- -) Analytical results using the conditions of the experiment $;(\bullet) E_{2}$, experimental measurements at $R e_{N L}=4.8 ;(\boldsymbol{-})$ $N_{2}$, numerical simulation $R e_{N L}=4.91 \ldots \ldots \ldots \ldots \ldots$

FIG. 6 (color online) Variation of the normalized axial streaming velocity component along the central axis of the guide. (- -) Analytical results using the conditions of the experiment $;(\bullet) E_{3}$, experimental measurements at $R e_{N L}=7.7 ;(\boldsymbol{-})$ $N_{3}$, numerical simulation at $R e_{N L}=7.93 \ldots \ldots \ldots \ldots$

FIG. 7 (color online) Variation of the normalized axial streaming velocity component along the central axis of the guide. (- -) Analytical results using the conditions of the experiment $;(\bullet) E_{4}$, experimental measurements at $R e_{N L}=14.6 ;(-)$ $N_{4}$, numerical simulation at $R e_{N L}=15.2 \ldots \ldots \ldots \ldots \ldots \ldots \ldots$ 
FIG. 8 (color online) Variation of the normalized axial streaming velocity component along the central axis of the guide. (- -) Analytical results using the conditions of the experiment $;(\bullet) E_{5}$, experimental measurements at $R e_{N L}=31.8 ;(-)$ $N_{5}$, numerical simulation at $R e_{N L}=38.6 \ldots \ldots \ldots \ldots \ldots$

FIG. 9 Streamlines of the streaming flow, from numerical simulation at $R e_{N L}=38.6$. 23

FIG. 10 (color online) Numerical simulations: variation of the normalized axial streaming velocity component along the central axis of the guide for several values of $R e_{N L} \ldots \ldots \ldots \ldots \ldots \ldots \ldots \ldots \ldots \ldots \ldots$

FIG. 11 (color online) Distribution of the harmonics along the guide. (a) Numerical simulation $N_{5}$; (b) Experimental study $E_{5} \ldots \ldots \ldots \ldots$

FIG. 12 (color online) Streaming velocity profile along the axis of the guide at $(R-$ $r) \approx 3 \delta_{\nu}$, for $R e_{N L}=38.6$. (-) Analytical solution ; (-.-) Streaming velocity from analytical superposition of frequency components of the numerically calculated acoustic velocity; (- -) Numerical simulation. . . . . . . . . . . . . 